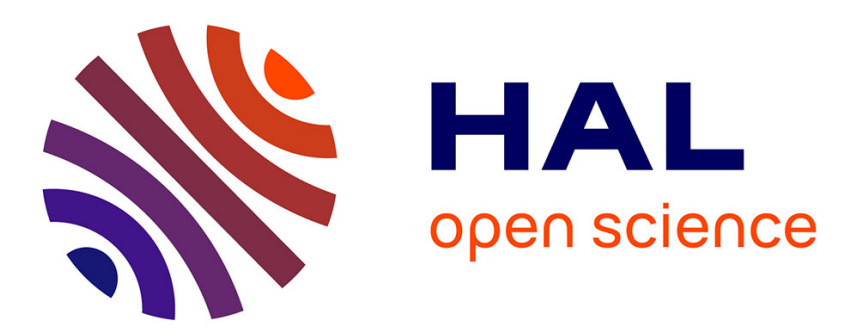

\title{
Les femmes dirigeantes de PME ont-elles des pratiques de RSE spécifiques ?
}

Agnès Paradas, Caroline Debray, Christophe Revelli, Jean-Marie Courrent

\section{To cite this version:}

Agnès Paradas, Caroline Debray, Christophe Revelli, Jean-Marie Courrent. Les femmes dirigeantes de PME ont-elles des pratiques de RSE spécifiques ?. Recherches en sciences de gestion, 2013, 97 (4), pp.187. 10.3917/resg.097.0187 . hal-02438052

\section{HAL Id: hal-02438052 https://hal.science/hal-02438052}

Submitted on 14 Jan 2020

HAL is a multi-disciplinary open access archive for the deposit and dissemination of scientific research documents, whether they are published or not. The documents may come from teaching and research institutions in France or abroad, or from public or private research centers.
L'archive ouverte pluridisciplinaire HAL, est destinée au dépôt et à la diffusion de documents scientifiques de niveau recherche, publiés ou non, émanant des établissements d'enseignement et de recherche français ou étrangers, des laboratoires publics ou privés. 


\section{LES FEMMES DIRIGEANTES DE PME ONT-ELLES DES PRATIQUES DE RSE SPéCIFIQUES?}

Agnès Paradas et al.

ISEOR | Recherches en Sciences de Gestion

2013/4 - N 97

pages 187207

ISSN 1634-7056

Article disponible en ligne l'adresse:

http://www.cairn.info/revue-recherches-en-sciences-de-gestion-2013-4-page-187.htm

Pour citer cet article :

Paradas Agnès et al., Les femmes dirigeantes de PME ont-elles des pratiques de RSE spécifiques ?,

Recherches en Sciences de Gestion, 2013/4 № 97, p. 187-207.

Distribution ectronique Cairn.info pour ISEOR.

ISEOR. Tous droits rerv pour tous pays.

La reproduction ou reprentation de cet article, notamment par photocopie, n'est autoris que dans les limites des conditions gales d'utilisation du site ou, le cas hnt, des conditions gales de la licence souscrite par votre ablissement. Toute autre reproduction ou reprentation, en tout ou partie, sous quelque forme et de quelque manie que ce soit, est interdite sauf accord prlable et rit de l'iteur, en dehors des cas prus par la lislation en vigueur en France. II est prisque son stockage dans une base de donns est alement interdit. 
revue Recherches en Sciences de Gestion-Management Sciences-Ciencias de Gestión, n97, p. 187 à 207

\title{
Les femmes dirigeantes de PME ont-elles des pratiques de RSE spécifiques ? $^{1}$
}

\author{
Agnès Paradas \\ Maître de Conférences - HDR \\ MRM Montpellier \\ Caroline Debray \\ Maître de Conférences \\ MRM Montpellier \\ Christophe Revelli \\ Professeur Assistant \\ KEDGE Business School \\ Jean-Marie Courrent \\ Maître de Conférences - HDR \\ MRM Montpellier
}

Cette recherche est effectuée dans le cadre du LabEx Entreprendre et du programme ANR GEODD, Université de Montpellier.

Cet article présente tout d'abord une revue de littérature sur les différences de comportements constatées entre hommes et femmes dans la société et dans les entreprises. Cette revue décrit également le

1. Cet article a été présenté sous la forme d'une communication au CIFEPME, Brest, 2012. 
cadre plus précis des pratiques socialement responsables développées par les dirigeantes. Il est ensuite proposé, à partir d'une enquête exploratoire auprès de 46 entrepreneurs également répartis entre hommes et femmes, d'identifier d'éventuelles différences dans les pratiques socialement responsables. Les résultats ne permettent pas de trancher, mais certaines pistes peuvent être envisagées.

Mots-clés : RSE, PME, Entrepreneuriat féminin, Développement durable.

This paper first presents a literature review on the different behaviors observed between men and women in society and companies. This review also describes the more specific framework of socially responsible practices developed by female leaders. In a second time, we propose, from an exploratory survey among 46 entrepreneurs equally divided between men and women, to identify possible differences in socially responsible practices. The results are inconclusive, but some tracks can be considered.

Key-words: CSR, Small Businesses, Women's Entrepreneurship, Sustainable Development.

Este artículo primero presenta una revisión bibliográfica sobre las diferencias existentes entre el comportamiento de los hombres y de las mujeres en la sociedad y en los negocios. Esta revisión también describe las prácticas de RSE de las mujeres empresarias. Se propone después una encuesta exploratoria de 46 líderes divididos en partes iguales entre hombres y mujeres, para identificar las posibles diferencias en las prácticas socialmente responsables. Los resultados no son concluyentes, pero algunas pistas pueden ser consideradas.

Palabras claves: Responsabilidad Social Empresarial, pequeñas empresas, mujeres empresarias, Desarrollo Sostenible. 


\section{Introduction}

La Responsabilité Sociale des PME (RSPME) suscite un intérêt croissant des différents acteurs économiques. Elle peut être définie comme l'application des principes du développement durable ${ }^{2}$ dans la stratégie des organisations. Elle défend également l'idée d'une mise en action volontaire de la part du dirigeant, qui joue un rôle central dans les petites structures. Ainsi, même si les déterminants de l'implication peuvent relever de différentes sources plus ou moins contraintes, le dirigeant a un rôle majeur dans la responsabilité sociale de son entreprise (Paradas, 2011 ).

Mais les recherches se heurtent à l'hétérogénéité des entrepreneurs et à la difficulté de cerner et de prescrire leurs comportements, leurs attitudes, leurs valeurs. Une façon d'y parvenir est d'observer une catégorie particulière, celle des entrepreneures. Parler de catégorie particulière est déjà une hypothèse. Mais observer les femmes entrepreneurs en sciences de gestion semble également constituer un apport en soi. La population des femmes entrepreneures est encore largement sous-étudiée. Seuls 6 à $7 \%$ en moyenne des études publiées depuis 1994 dans le Top 8 des Journaux référencés en Entrepreneuriat concernent ce sujet (De Bruin et al., 2006).

Trois intérêts permettent de justifier la pertinence d'une recherche sur les femmes entrepreneures et la RSE de leur entreprise.

Le premier intérêt renvoie à l'orientation plus féminine des valeurs de la société (Marsan, 2007, Luyckx, 2002) et à la croissance de l'entrepreneuriat féminin. Les entrepreneures incarnent environ $30 \%$ des entrepreneurs, sont souvent cantonnées à des secteurs particuliers (services et commerce) et plutôt actives dans les plus petites structures. Elles semblent sous-représentées en Europe (Drion, 2006 ; Revue Industrie, 2008). Toutefois, les modèles anglo-saxons montrent que les femmes sont davantage représentées et en forte croissance dans le domaine de la direction de PME (Carrier et al., 2006 ; Vier Machado et Rouleau, 2002).

Le deuxième intérêt tient à l'existence de différences constatées dans le mode de management et les comportements des

2. La définition de la Communauté Européenne dans son livre vert de 2001 englobe les dimensions sociale, environnementale et économique de long terme ainsi que la problématique des parties prenantes. En octobre 2011, la CE fait figurer plus explicitement les droits de l'homme dans le champ de la RSE et positionne la responsabilité des entreprises vis-à-vis des effets qu'elles exercent sur la société (Courrent, 2012). Rappelons également que le sommet de Rio 1992 énonce comme premier principe de sa déclaration : les êtres humains sont au centre des préoccupations relatives au développement durable. 
entrepreneures, comparativement aux hommes entrepreneurs. La synthèse de 25 ans d'études sur l'entrepreneuriat féminin proposée par Carrier et al. (2006) permet de distinguer les conditions d'exercice entrepreneurial selon le sexe.

Le troisième intérêt concerne l'apparente proximité des valeurs féminines et des valeurs du développement durable. Une autre hypothèse s'impose alors, celle de l'association entre sexe féminin et valeurs féminines. Sans entrer dans ce débat, et cela pouvant constituer une limite de notre travail, nous assimilerons volontairement femmes et valeurs féminines. L'entrepreneuriat féminin afficherait des dimensions plus «humaines » et moins «économiques» que l'entrepreneuriat masculin. Les femmes entrepreneures adopteraient des valeurs proches du développement durable. Les résultats de l'enquête récente de Saint-Pierre et al. (2011) permettent ainsi de conclure que les femmes ont une attitude plus favorable au développement durable que les hommes.

L'objectif de cet article est d'explorer une question très rarement étudiée ${ }^{3}$, celle des différences entre hommes et femmes entrepreneurs dans leurs pratiques socialement responsables. Il s'intègre dans une recherche plus ambitieuse tendant à identifier d'éventuelles distorsions de représentations à l'origine de ces comportements.

Pour cette exploration, des données ont été traitées par analyse factorielle des correspondances multiples (AFCM). Elles proviennent de l'observation de 25 variables liées aux pratiques environnementales, sociales et sociétales, auprès d'un échantillon de 46 dirigeants de PME, dont la moitié est constituée de femmes.

Cet article présentera dans une première partie des éléments de discussion autour des différences pouvant être observées entre les hommes et les femmes ainsi que la méthodologie choisie pour l'enquête. Une deuxième partie permettra de présenter les résultats de cette étude et d'engager une discussion.

\section{1. - Cadre conceptuel et méthodologie}

Les travaux relatifs aux distorsions de valeurs et de comportements entre hommes et femmes suscitent des controverses. Une enquête exploratoire, en ce sens, trouve toute sa justification.

\footnotetext{
3. Après une revue de la littérature, il apparaît que la conjonction DD ou RSE / femmes / PME n'ait fait l'objet que de très rares travaux. L'article de St-Pierre et al. (2011) est le plus proche de nos préoccupations, mais étudie davantage les objectifs et la performance en matière de DD que les pratiques déclarées.
} 


\subsection{Les dirigeantes et la RSE}

\subsubsection{Identification de sources de différences}

Les entrepreneurs sont avant tout des hommes et des femmes, et cela peut avoir d'autant plus d'importance dans les PME, fortement marquées par les profils de leurs dirigeants.

\subsubsection{Origines physiologiques et psychosociologiques}

Les recherches dans le domaine sont très nombreuses et sortent du champ des sciences de gestion. Certains travaux récents peuvent toutefois être cités pour illustrer les discussions actuelles. Catherine Vidal (2006) montre dans ses recherches sur le cerveau que sur 1000 études, seulement $3 \%$ ont indiqué une différence entre les sexes. $88 \%$ des connexions se faisant après la naissance, c'est l'interaction avec l'environnement qui influence ces connexions. Elle évacue ainsi l'hypothèse biologique de différenciation entre les sexes. Cette position est relayée par Marsan (2007) qui indique que toutes les théories affirmant la différence structurelle et physiologique des cerveaux entre les hommes et les femmes sont fausses. Nous avons les mêmes dispositions biologiques et neurologiques à la naissance que nous soyons homme ou femme. D'après Vidal (2006), l'extension du cortex permet chez l'être humain d'échapper au déterminisme des gènes et des hormones. Cela met au centre des réflexions l'importance des facteurs individuels, sociaux et culturels. Ce sont essentiellement la culture, l'éducation et les stimulations biologiques qui vont avoir une influence sur le développement de notre cerveau, de notre pensée et de nos capacités d'apprentissage, de connaissance et donc notre intelligence (Marsan, 2007).

Hormis l'influence des hormones sexuelles ${ }^{4}$, deux autres facteurs fondamentaux peuvent pourtant expliquer certaines différences.

Le premier est celui de la maternité. Le rôle de mère impliquerait des représentations et des comportements différents. La fonction reproductive conduirait ainsi à une nature qui rendrait les femmes plus sensibles au devenir et à la vie des générations futures (Zuinen, 2002) et même pour les femmes sans enfant. Le lien entre le rôle de mère et le rôle de gestionnaire est un élément essentiel de leurs représentations (Vier Machado et Rouleau, 2002). La fonction maternelle induirait

4. Dans le Figaro du 6 février 2012, J-L Nothias fait état d'une expérience réalisée à l'University College of London à propos de la testostérone sur des femmes, ce stéroïde nous rendant «moins coopératif avec les autres et plus égocentrique ». 
certaines difficultés dans l'exercice entrepreneurial et un investissement moindre en temps consacré à l'entreprise (St-Pierre et al., 2011). Toutefois pour certains auteurs la conciliation travailfamille n'a pas d'influence sur la performance (Carrier et al., 2006).

Le second facteur est celui de la fonction « historique » de maîtresse de maison. Selon Vidal (2006), l'appartenance des hommes à la sphère publique et des femmes à la sphère domestique, oriente les uns vers l'espace et les autres vers le langage et la transmission des savoirs. Cela influence l'estime de soi, souvent inférieure chez les femmes. Mais la culture égalitaire effacerait les écarts. Et les différences semblent s'atténuer avec l'arrivée des jeunes générations. La fonction de maîtresse de maison donnerait aussi aux femmes une vision plus intégrée des relations entre les êtres humains, leurs activités de consommation et leur environnement naturel (Zuinen, 2002). La relation au bénévolat, tout comme l'inclinaison vers le care semblent également influencées par cette fonction (Drion, 2006 ; Zouiten et Levy-Tadjine, 2005, Degavre, 2005). D'après Drion (2006), cela porte en germe la critique des modèles économiques classiques et permettrait de positionner les femmes comme actrices d'un nouveau type de développement.

Les valeurs féminines, portées par la nature reproductive et la fonction ménagère seraient plutôt de l'ordre de la solidarité, du don, du care, de la recherche de consensus et de l'attention à l'autre. Et même s'il est réducteur d'opposer systématiquement les valeurs masculines aux valeurs féminines, il est intéressant de se placer dans le contexte de leur complémentarité. L'influence des valeurs féminines dans la société amènerait à une nouvelle façon de voir le monde et d'agir sur lui, à une recherche de coopération plus que de compétition. Au sein de l'entreprise, certains parallèles peuvent être faits.

\subsubsection{Les sources socio-économiques}

Des questions émergent par exemple au sujet de la compatibilité des valeurs féminines et de la notion de profit. La femme, dans ce contexte, peut féconder l'esprit des hommes dans un sens favorable à d'autres valeurs que le profit (Rousseau, 2007). Lambrecht et al. (2003) ou Zouiten et Lévy-Tadjine (2005) rejoignent ce point de vue en observant des entrepreneures plus intéressées par leur contribution et la valorisation des besoins sociaux que par la recherche d'un bénéfice personnel, d'un statut ou d'argent. Il s'agit cependant d'être prudent face à cette relation au profit, car nombre d'observateurs de 
PME montrent que les dirigeants, sans distinction de sexe, ont souvent d'autres motivations que le profit dans leur activité entrepreneuriale (GREPME, 2007, Klein and Kellermanns, 2008).

L'observation des femmes entrepreneures permet de mettre en évidence certaines constantes dans les différences de gestion entre hommes et femmes. Selon plusieurs auteurs cités par Vier-Machado et Rouleau (2002), la gestion féminine serait caractérisée par une pratique du pouvoir plus horizontale et participative, par une recherche de satisfaction des proches et des employés et par un intérêt pour la responsabilité sociale. St-Pierre et al. (2011) évoquent également des études comparatives entre hommes et femmes entrepreneurs qui montrent que les entrepreneures privilégient l'épanouissement et la maîtrise de soi, la créativité et la recherche de qualité.

Certaines analyses récentes suggèrent que l'entrepreneuriat est «en soi une mentalité et une expérience masculine» (De Bruin et al., 2006, p. 586). Ce qui signifierait selon Achtenhagen et Welter (2004) que les chercheurs et la société en général associent plus aisément les actions entrepreneuriales aux hommes qu'aux femmes (cité par De Bruin et al., 2006, p. 587).

Selon Ahl (2006), la féminité serait-elle alors construite comme le contraire de l'esprit d'entreprise ? L'auteure a dressé une liste des mots opposés au mot entrepreneur en utilisant un dictionnaire d'antonymes et essayé de faire correspondre le mot entrepreneur à des mots de féminité de Bem. L'auteure constate que l'entrepreneur est représenté de manière positive, alors que les mots opposés sont largement négatifs. La conclusion est que l'entrepreneur est un concept masculin, c'est-à-dire qu'il n'est pas neutre.

La féminisation des valeurs de la société traduit une progression, chez les hommes comme chez les femmes, de l'importance attachée à l'esthétique, aux émotions, à la coopération entre individus et à la prise en compte du long terme. Cela explique en partie le développement des réseaux sociaux et le souci croissant de développement durable. La relation entre entrepreneure et développement durable va donc maintenant être analysée.

\subsubsection{Les entrepreneures face à la $\mathrm{RSE}$}

Les recherches dans le domaine sont rares, mais certains éléments d'études peuvent illustrer notre propos. 


\subsubsection{Relations avec les parties prenantes}

Les femmes semblent posséder une plus grande capacité d'interaction avec les parties prenantes. Plusieurs recherches montrent une attention particulière des entrepreneures envers autrui et une sensibilité à ce que pensent les autres (St-Pierre et al., 2011). Cette attention est accompagnée de représentations poussant davantage les femmes à faire des compromis avec les clients, les employés et dans les relations interpersonnelles (Vier-Machado et Rouleau, 2002). Celles-ci sont caractérisées par une vision différente du pouvoir. La dimension participative et interactive des relations (Carrier et al., 2006), la volonté d'impliquer les différentes parties en présence rendent les modalités des actions envers les parties prenantes différentes de celles des hommes (Carter et al., 2007). L'exercice du leadership serait plus démocratique et relationnel et les entrepreneures porteraient une attention particulière à l'équité et au bien-être de la communauté (St-Pierre et al., 2011). A cela se rajoute une recherche d'effectivité et la présence d'affectivité, plus que d'efficience et d'efficacité (Mione, 2004). Ainsi, citant une étude de 1992 réalisée par Mc Grath et Mc Millan auprès de 700 entrepreneurs de pays différents, Vier-Machado et Rouleau (2002) concluent que la représentation du succès pour les entrepreneures est définie par rapport à la satisfaction des autres personnes, plus que par rapport à une conception de la personne isolée.

Une place particulière doit être faite à la relation des femmes avec les réseaux. Pourquoi, alors que les femmes sont enclines à favoriser les relations, le constat reste celui d'une sous-représentation relative des femmes dans les réseaux, pointée par différents auteurs (Carrier et al., 2006, St-Pierre et al., 2011, Géraudel et al., 2009) ? Les réseaux sont surtout mobilisés lorsqu'ils sont en lien direct avec l'activité ou le domaine de formation et ils sont davantage utilisés pour le conseil que pour le contact ou la recherche d'opportunités. L'étude de Cornet et al. (2005) auprès de 288 entrepreneures fait quand même état de 58,3\% de membres d'un ou de plusieurs réseaux d'affaires. La proximité avec l'activité et la formation est également constatée et les principaux freins évoqués sont, dans l'ordre, le manque de temps, le manque d'informations sur les réseaux existants ou le manque d'intérêt. 


\subsubsection{Les femmes face au triptyque environnement - social - économie}

De manière générale, il semble que les femmes montrent un plus fort intérêt pour des objectifs socio-environnementaux que les hommes (St-Pierre et al., 2011).

Concernant les relations avec les salariés, Fouquet (2005) indique dans une étude élargie auprès d'entrepreneures, que $90 \%$ d'entre elles voient des divergences dans le style de relations personnelles. Par ailleurs, les résultats d'un sondage du cabinet de conseil McKinsey \& Company (4ème rapport Women Matter 2010) montrent que la capacité «d'attente et de reconnaissance» des femmes entrepreneures ainsi que leur tendance à stimuler «l'inspiration » de leurs subalternes auraient joué un rôle déterminant dans le maintien des performances de leur entreprise. Selon les analyses de littérature de Carrier et al., (2006) ou de St-Pierre et al. (2011), les femmes présenteraient des différences dans leurs styles de gestion. L'approche des entrepreneures serait plus personnelle, plus maternelle et plus proche des préoccupations des salariés. On retrouve la tendance à partager le pouvoir dans une démarche plus souvent participative et interactive. Si certaines qualités liées à l'estime de soi ou à la reconnaissance d'un rôle féminin d'entrepreneuriat leur manquent, elles misent davantage sur l'écoute, l'encouragement et délèguent plus facilement. Cette capacité d'écoute et cette recherche du consensus, ainsi que l'attention envers les autres, sont en effet considérées par les femmes elles-mêmes comme des aspects de management typiquement féminins (Fouquet, 2005).

Entre le social et l'environnemental, le sociétal tient également une place importante dans le développement durable. Certains chiffres interpellent. Par exemple, une étude de la Harvard Business School montre un lien fort entre la présence de femmes dans la gouvernance et l'exercice de la philanthropie et des causes humanitaires. En 2007, les entreprises qui avaient au moins 3 administratrices ont donné 28 fois plus que celles où ne siègent que des hommes. Une femme en plus dans le CA et c'est 1.7 millions d'euros supplémentaires. Si la femme est PDG ce sont 5.7 millions d'euros supplémentaires.

Enfin, certaines informations sont à relever dans le domaine de la relation des femmes à l'environnement, au point que certains parlent même d'écoféminisme (Pandey, 2010). St-Pierre et al., (2011) concluent que les femmes accordent une importance significativement plus grande que les hommes à la protection de l'environnement par des politiques internes ainsi que par le choix de fournisseurs selon leurs bonnes pratiques environnementales. Le déterminisme social 
évoqué plus haut d'une femme responsable de son foyer influence certainement ces constats. Partout dans le monde, les femmes sont expertes des produits de consommation. Parce qu'elles sont plus sensibles à l'impact qu'elles peuvent avoir sur la santé et le bien-être de leur famille au quotidien, les femmes seraient plus soucieuses que les hommes de l'environnement. Plusieurs auteurs pointent la moindre empreinte des femmes sur la planète (Drion, 2006, Johnsson-Latham, 2006), les femmes utilisant une surface inférieure aux hommes pour produire ce qu'elles consomment et absorber ce qu'elles rejettent. Cela peut avoir une influence forte sur les choix de management et les orientations environnementales.

Le dernier élément constituant du développement durable concerne la performance durable. Elle est un pilier essentiel de la réussite d'un projet soutenable, même si, comme nous l'avons vu, la performance peut relever de réalités multiformes et ne se cantonne pas uniquement au profit. La durabilité s'oppose d'ailleurs à une recherche de maximisation du profit à court terme. La performance peut par exemple se décliner en performance socio-environnementale.

Dans ce cadre, les résultats obtenus par St-Pierre et al., (2011) montrent que tous les indicateurs d'une bonne performance au plan socio-environnemental sont supérieurs pour les femmes. Une autre étude sur des statistiques canadiennes réalisée par Robichaud et Mc Graw et citée par Carrier et al., (2006) mérite également notre attention. En effet, entre 1991 et 1994, les entreprises dirigées par des femmes ont créé 4 fois plus d'emplois que la moyenne canadienne. Et selon le rapport Women Matter 2010 (op.cit), la présence de femmes dans les comités d'administration favoriserait l'amélioration des performances.

Concernant plus particulièrement les résultats financiers, les débats sont engagés (St-Pierre et al., 2011 ; Carrier et al., 2006). Il ne semblerait pas y avoir de différence significative entre les aspirations féminines ou masculines. Mais tout cela est à nuancer en fonction des secteurs d'activité, de l'âge des personnes ou de la taille des entreprises. Deux profils semblent apparaître : d'une part des femmes qui ont intégré des principes de marché portés par des valeurs masculines de compétition et de croissance, à la recherche d'une performance maximale et de la valorisation d'objectifs économiques ; d'un autre coté des femmes qui privilégient d'autres valeurs, plus «féminines » comme celles évoquées dans notre présentation. Si les femmes ont eu tendance, à la place où elles étaient et avec leur nature "vénusienne", à incarner de telles valeurs, cela ne signifie pas que toutes les incarnent, ni qu'elles en aient l'exclusivité (Rousseau, 2007). 
Ces différentes observations indiquent toutefois que les entrepreneures risquent de développer des pratiques différentes des entrepreneurs en matière de RSE. Pour cela, une exploration peut être utile.

\subsection{Choix méthodologiques}

La méthodologie choisie doit permettre de percevoir, dans une perspective exploratoire, des correspondances et proximités ${ }^{5}$ entre la variable sexe et les pratiques déclarées en matière de RSE.

Le questionnaire dont sont tirées les données a été élaboré par une équipe de chercheurs à partir de modèles théoriques et d'observations empiriques. Seules trois questions ont été directement retenues au sein de ce questionnaire traité en 2012. Toutefois, d'autres questions relatives aux variables contingentes ont également été utilisées pour construire l'échantillon.

Chacune des trois questions contient entre 8 et 9 propositions traitées par échelles de Likert à 5 points, soit environ 40 modalités par question. Afin de simplifier les interprétations dans cette analyse exploratoire, les items 1 et 2 (faiblement ou pas $d u$ tout) ont été regroupés en une seule modalité «-», le 3 en «=» et les 4 et 5 (plutôt ou tout à fait) en «+». Les non-réponses ont également été prises en compte («?»).

Les trois groupes de variables retenus renvoient aux pratiques des dirigeants vis-à-vis de l'environnement, des questions sociales internes et de la communauté . $^{\text {. }}$.

Une fois la base de données épurée, les femmes dirigeantes étaient au nombre de 23 dans l'échantillon. Compte tenu des contingences observées dans les recherches (Carrier et al., 2006), il a été décidé de choisir 23 hommes dirigeants présentant une proximité en termes d'activité, d'effectif et d'ancienneté de l'entreprise ainsi que de formation des dirigeants. La proximité géographique (France) concerne toutes les unités. L'échantillon observé contient donc au final 46 PME (répartition «normale» depuis les TPE aux ME) d'activités très variables.

Les traitements statistiques choisis sont des traitées selon les recommandations d'Evrard et al. (2003). Au-delà de l'intérêt que de telles analyses présentent en matière de comparaisons, elles permettent également de percevoir des groupes et de construire certaines typologies de réponses. Pour cela, les modalités de réponses

5. Voir par exemple le chapitre 6 de Gavard-Perret et al. (2008).

6. Voir annexe 2. 
ont été traitées simultanément et la variable «sexe » a été projetée sur le plan factoriel ${ }^{7}$. L'AFCM, à visée descriptive, sera complétée par des tests de régression explicatifs en vue de renforcer notre analyse.

Il s'agit de traiter la globalité des résultats obtenus dans une perspective d'approche combinée de trois groupes de pratiques, et non de les traiter de manière séparée.

\section{2. - Résultats et discussion}

\subsection{Les résultats}

Une fois les variables décrites traitées par le biais de l'AFCM, il apparaît que les deux premiers axes contribuent de manière satisfaisante à la description des données, puisqu'ils représentent plus de $61 \%$ en pourcentage cumulé d'inertie ajustée de Benzécri. L'axe 1 à lui seul représente $44,36 \%$ d'inertie et l'axe 2 en représente $16,79 \%$.

Un tri a été fait parmi les modalités afin de ne conserver que les valeurs qui respectent les seuils nécessaires à une interprétation acceptable selon le cadre méthodologique défini. Elles sont appelées modalités contributives.

Le premier tri selon les axes donne les résultats présentés dans le tableau 1 (annexe 1). L'interprétation de ce tableau permet d'opposer les pratiques plutôt masculines (projection sur les coordonnées positives des axes 1 et 2) ou féminines (projection sur les coordonnées négatives des axes 1 et 2) en fonction des coordonnées sur les axes. Sur l'axe 1, l'opposition se forme entre des non-réponses plutôt portées par les hommes et des réponses liées à des pratiques négatives plutôt portées par les femmes. Sur l'axe 2, les réponses à caractère négatif sont associées aux hommes tandis que les réponses liées à des pratiques positives sont assimilées aux femmes.

Deux points majeurs semblent se distinguer sur l'axe $1:$ «le recrutement de salariés handicapés au-delà des obligations », suscitant les seules réponses à caractère positif dans le groupe de non-réponses attribuables globalement aux hommes, et «l'approvisionnement

\footnotetext{
7. Dans l'AFCM, il s'agit de distinguer variables actives et variables supplémentaires. Les variables actives contribuent à l'inertie des axes au contraire des variables supplémentaires (qui sont projetées à titre illustratif sur le plan factoriel). Dans le cadre de cette recherche, la variable « sexe » est considérée comme supplémentaire, car nous souhaitons seulement observer son positionnement par rapport aux pratiques environnementales, sociales ou sociétales au sein de typologies.
} 
prioritaire au niveau local ou régional » engendrant les seules réponses à caractère positif parmi les réponses négatives, globalement attribuables aux femmes sur cet axe.

Toutefois, ce tableau rend compte de la répartition des variables selon chaque axe, mais ne permet pas de donner une interprétation englobant les axes 1 et 2 simultanément. La répartition des modalités de variables rend difficile l'extraction de typologies réellement évidentes.

L'observation du plan factoriel obtenu et le repérage des seules variables contributives permettent d'ailleurs de faire émerger des interprétations supplémentaires concernant les typologies. Sur le plan, quatre groupes de réponses - pratiques positives, négatives, moyennes et sans réponses - apparaissent visuellement de manière assez naturelle. Les modalités qui sont regroupées au centre du plan sont les moins contributives. Or c'est également le cas pour les variables supplémentaires hommes et femmes. Cela induit une certaine prudence dans les interprétations qui tendraient à distinguer les pratiques masculines et féminines.

Il apparaît aussi que les réponses sont très homogènes dans chaque questionnaire. Les dirigeant(e)s ont respectivement proposé des réponses positives, négatives ou neutres, ou bien un nombre important de non-réponses. Les pratiques, si elles sont (ou pas) développées dans le domaine environnemental, le sont (ou pas) également dans les domaines sociaux ou sociétaux. Même si ce résultat ne répond pas directement aux interrogations, il constitue un apport.

Par contre, dans le cadre de cette recherche distinguant les hommes et les femmes entrepreneurs, le seul axe 1 (inertie de 44,36\%) conduit à rapprocher les pratiques négatives(-) des femmes et les pratiques positives(+) ou les sans réponses( ?) des hommes, alors que l'axe $2(16,79 \%)$ donne le résultat inverse. Ainsi, cette analyse ne permet pas de conclusions quant à des différences visibles entre hommes et femmes dans l'exercice de leurs pratiques.

Devant cette difficulté et afin de renforcer l'analyse descriptive réalisée en première instance, des analyses explicatives ont été réalisées. Ainsi, plusieurs ANOVA uni-variées ont été réalisées sur l'échantillon global de 46 personnes. Afin de ne pas exclure les nonréponses des traitements, ce qui aurait pour effet de supprimer un nombre conséquent d'observations de l'analyse, une modalité supplémentaire " non-réponse $=0$ » a été créée. Il est considéré qu'un non-répondant à la question posée sur une échelle d'intervalle ne prend pas en compte les principes présentés. 
L'intérêt est de comprendre si le sexe influence les pratiques environnementales, sociales ou sociétales.

Dans le tableau 2, il apparaît que de manière globale, la variable « sexe » n'influence pas les pratiques RSE des entrepreneurs, puisque le $\mathrm{R}^{2}$ est très faible $(0,007)$ et le test $\mathrm{F}$ de Fisher non significatif. Ce tableau nous informe seulement sur la validité statistique du modèle dans son ensemble.

Tableau 2 - Analyse de variance globale

\begin{tabular}{|l|l|l|l|l|l|}
\hline Source & DDL & $\begin{array}{l}\text { Somme des } \\
\text { carrés }\end{array}$ & $\begin{array}{l}\text { Carrés } \\
\text { moyens }\end{array}$ & F & p-value \\
\hline Facteur & 1 & 0,783 & 0,783 & 0,291 & 0,592 \\
\hline Résidus & 44 & 118,174 & 2,686 & & \\
\hline $\begin{array}{l}\text { Total } \\
\text { corrigé }\end{array}$ & 45 & 118,957 & & & \\
\hline
\end{tabular}

Il s'agit maintenant d'observer les différences entre hommes et femmes sur les pratiques détaillées, via les tests $\mathrm{T}$ de Student de différences de moyennes issus des ANOVA. Utilisant un petit échantillon (46 sondés), il est nécessaire de renforcer l'analyse en appliquant le test non paramétrique U de Mann-Whitney. Ce test est l'alternative la plus utile au test paramétrique $\mathrm{T}$ lorsque les échantillons étudiés sont de petite taille. Les résultats issus de ces tests sont présentés dans le tableau 3 (annexe 2). Selon eux, il n'existe pas de différence significative entre hommes et femmes dans la prise en compte des pratiques RSE. Ainsi, les pratiques environnementales, sociales et communautaires ne sont pas influencées par le sexe de l'entrepreneur dans la grande majorité des pratiques mises en avant dans l'enquête.

Seulement deux types de pratiques divergent : les hommes ont davantage tendance à recruter des salariés handicapés par rapport aux femmes (significatifs dans le cas du test $\mathrm{T}$ de Student), tandis que les femmes sont plus ancrées dans le concept d'écoconception en étant plus attentives à l'impact de leur production sur l'environnement (significatifs dans le cas du $\mathrm{T}$ de Student et du test U de MannWhitney).

\subsection{Discussion et conclusion}

La conclusion principale de cette recherche est que les différentes analyses laissent à penser que les orientations entre 
hommes et femmes entrepreneurs en matière de pratiques RSE ne sont pas saillantes.

L'étude de St-Pierre et al. (2011) montre que les femmes favorisent davantage que les hommes les dimensions liées au développement durable dans leur entreprise. Mais cet article présente une recherche comparative entre les objectifs liés au développement durable des hommes et femmes entrepreneurs, alors que la recherche porte ici sur les pratiques déclarées des dirigeants.

$\mathrm{Si}$ la relation entre femmes et valeurs éthiques a été explorée dans un certain nombre de recherches (un peu plus d'une centaine identifiées à ce jour), la plupart s'éloignent du contexte précis dans lequel se situe cette recherche, soit en traitant de publics différents comme les étudiants ou les cadres, soit en étudiant davantage les attitudes, déclarations ou intentions. Et les résultats sont très variables. Dutta et Banerjee (2011) proposent par exemple une recherche concernant les différences de sexe en matière de pratiques éthiques des affaires. Ils concluent à des pratiques des entrepreneures inférieures à la catégorie de référence des entrepreneurs de sexe masculin, indiquant qu'ils contredisent ainsi «la sagesse conventionnelle» (p. 217). Leur travail portant sur le cas particulier de l'éthique dans le domaine social, ils constatent que les femmes observées dirigent davantage les plus petites entreprises de leur échantillon et sont moins contraintes par les lois, ont moins suivi de formations officielles et sont donc moins sensibilisées. Les auteurs recensent également huit travaux (entre 1984 et 2008) aux problématiques proches qui présentent des résultats contradictoires. Plusieurs références signalent la supériorité des femmes en matière de pratiques éthiques, en particulier dans les petites entreprises. Mais d'autres concluent également à l'absence de différence.

Dans le cadre de la présente enquête, l'augmentation du nombre des unités statistiques et la recherche élargie aux attitudes semblent nécessaire pour pouvoir affirmer (ou non) une différence.

Nos analyses permettent également l'évocation de trois cas particuliers.

Les deux traitements statistiques indiquent une tendance supérieure des hommes à « recruter des salariés handicapés » au-delà des obligations. La littérature ne fournit pas, à notre connaissance, de clé d'interprétation possible de cette conclusion.

L'AFCM indique que sur l'axe 1, «l'approvisionnement prioritaire au niveau local ou régional » est la seule réponse positive parmi les réponses négatives. Une explication peut être trouvée dans le fait que cette pratique puisse tout autant poursuivre des objectifs purement stratégiques ou de nécessité pour l'entreprise et ne pas 
s'insérer, alors, dans une démarche volontaire d'intégration dans la communauté ou de développement local. Il est intéressant de constater que les conclusions de l'enquête de St-Pierre et al. (2011) établissent également une situation particulière pour cet item. Les auteurs constatent dans leur étude une absence de motivation distincte (H/F) pour «la sélection des fournisseurs dans la région» alors que les autres objectifs socio-environnementaux montrent un plus fort intérêt pour les femmes.

Les tests de Student et de Mann-Whitney démontrent que les femmes sont plus ancrées dans le concept «d'écoconception », c'està-dire plus attentives à l'impact de leur production sur l'environnement. Cela rejoint en partie des éléments de la littérature présentée.

Enfin, les résultats obtenus ne sont pas dépendants des variables de contingence telles que l'activité, l'effectif, l'ancienneté, la situation géographique de l'entreprise et la formation des dirigeants. En effet, comme cela a été expliqué plus haut, les hommes et les femmes dirigeants choisis dans l'échantillon présentent des proximités pour tous ces facteurs et l'échantillon est varié. Or les travaux qui observent des différences de comportement des entrepreneurs et entrepreneures indiquent la plupart du temps une influence possible de facteurs (Carrier et al., 2006, Labelle et St-Pierre, 2010, Dutta et Banerjee, 2011) et en particulier de la taille des entreprises (St-Pierre et al., 2011). On peut ainsi se demander si le fait que ces variables soient neutralisées peut être une explication aux conclusions relativement peu marquées pour de nombreuses modalités.

Face à ces résultats, il semble évident que plusieurs approfondissements seront nécessaires. Ils peuvent renvoyer à un accroissement de la taille de l'échantillon. L'observation des «cas particuliers » concernant certaines modalités peut également donner lieu à des investigations plus en profondeur. Enfin, la dimension liée à la performance des entreprises en fonction de l'implication dans le développement durable devra également être traitée, car elle est absente des analyses présentées alors qu'elle tient une place majeure dans les pratiques socialement responsables.

Les recherches quantitatives élaborées sur le sujet donnent finalement des résultats assez contrastés. Cela peut être attribué à une limite essentielle des recherches quantitatives qui ne permettent pas de prendre en compte toutes les variables d'influence des pratiques RSE. Même si certaines de ces variables ont été neutralisées, d'autres pourraient jouer un rôle sans être identifiées. Une approche qualitative davantage orientée vers une recherche narrative serait alors très utile pour repérer ces éléments, ce que conforte un autre argument : récemment évoquée dans le Monde du 5 mars 2013, une méta-analyse 
de Sarah Saint-Michel affirme l'inexistence de différences de compétences de leadership entre hommes et femmes. Et ce que pointe également l'auteure est l'importance des représentations des différents acteurs (leaders et collaborateurs) qui interagissent en fonction de stéréotypes (les femmes renverraient l'image que l'on attend d'elles). Ces conclusions montrent ainsi la nécessité d'utiliser des méthodes d'investigation plus fines et plus approfondies, permettant d'identifier ces jeux d'acteurs.

Cet article est donc une première étape d'une recherche plus importante qui donnera lieu par la suite à un protocole d'élargissement des études quantitatives et d'études qualitatives, visant à améliorer la compréhension et le rôle des valeurs dans l'implication socialement responsable des dirigeant(e)s de PME.

\section{RÉFÉRENCES BIBLIOGRAPHIQUES}

AHL A., «Why Research on Women Entrepreneurs Needs New Direction », Entrepreneurship Theory and Practice, 30 (5), Sept, p. 595-621, 2006.

CARrier C., Julien P-A., Menvielle W., «Entrepreneuriat féminin : une synthèse des études des 25 dernières années », Gestion, 31 (2), p. 36-50, 2006.

CARTer S., Shaw E., Lam W., Wilson F., «Gender, entrepreneurship, and bank lending: the criteria and processes used by bank loan officers in assessing applications », Entrepreneurship Theory and Practice, 31(3), p. 427-444, 2007.

Cornet A., Constantinidis C., avec la collaboration de Asendei S., «Les femmes entrepreneures face à la formation, au financement et aux réseaux », Projet Diane, ULg, Egid, UCM, Liège, 2005.

COURRENT J-M. (dir.), RSE et DD en PME : comprendre pour agir, De Boeck, Collection Petites entreprises et entrepreneuriat, Avril, 2012.

Degavre F., «Enjeux du développement dans les contextes Nord : le rôle des femmes dans le care et la reproduction du lien social », Thèse de doctorat, ESPO/SPED, 2005.

De Bruin A., Brush C.G., Welter F., «Introduction to the special issue: towards building cumulative knowledge on women's entrepreneurship », Entrepreneurship Theory \& Practice, 30 (5), p. 585-593, sept, 2006.

DRION C., «Femmes et DD : vision d'avenir, entrepreneuriat et recommandations », Le monde selon les femmes, Collection Analyse \& Plaidoyer, 2006. 
DutTa S., BANERJEe S., «Ethical practices towards employees in small enterprises: a quantitative index », International Journal of Business Management \& Economic Research, 2(3), p. 205-221, 2011.

EVRARD Y, PRAS B., ROUX E., Market : Etudes et recherches en marketing, 3ème édition, Collection Gestion Supérieure, Dunod, Paris, 2003.

FouQuet A., «Les femmes chefs d'entreprise : le cas français », Travail, genre et société, $\mathrm{n}^{\circ} 13,2005$.

Gavard-Perret M-L., Gotteland D., HaOn C., Jolibert A., Méthodologie de la recherche, Pearson Education, 2008.

Géraudel M., Chauvet V., Chollet B., « Information returns and personal networks: does gender matter? », European Academy of Management Conference, Liverpool, May 11-14, 2009.

GREPME, Les PME : bilan et perspectives, sous la direction de Julien P.A., $2^{\circ}$ Edition, Economica, Paris, 1997.

JoHNSSON-LATHAM, G., «Initial study of lifestyles, consumption patterns, sustainable development and gender: do women leave a smaller ecological footprint than men?, Report from the Sweedish Ministy of Sustainable development. http://www.genderandenvironment.org/arcangel2/documentos/447.pdf, 2006.

Klein S., Kellermanns F., «Understanding the Noneconomic-Motivated Behaviour in Family Firms: An Introduction », Family Business Review, 21(2), p. 121-125, 2008.

LABELLE F., St-PIERRE J., « Les déterminants institutionnels, organisationnels et individuels de la sensibilité des PME au sujet du développement durable», Actes $d u \quad X^{\circ}$ Congrès International Francophone en Entrepreneuriat et PME (CIFPME), Bordeaux, 2010.

LAMBreChT J., Pirnay F., AMEdOdJI P., AOUNI Z., Entrepreneuriat féminin en Wallonie, Centre de Recherche PME et d'Entrepreneuriat - Université de Liège et Centre d'Etudes pour l'Entrepreneuriat - EHSAL - K.U. Brussel, 231 pages, 2003.

LUYCKX M., «Le rôle de l'expert : participer au ré-enchantement du monde », Reflets et perspectives de la vie économique 1/2002 (Tome XLI), p. 89-99, 2002.

MARSAN C., "Au-delà du masculin et du féminin », Cahiers de psychologie politique, numéro 11, 2007.

http://lodel.irevues.inist.fr/cahierspsychologiepolitique/index.php?id=581,

Mione A., "CIGALINE, entre affectivité, effectivité et efficacité, Bilan des cinq premières années de vie de l'unique Club d'Investissement pour la Gestion Alternative de l'Epargne Solidaire, au féminin », Communication au 7eme CIFPME, Montpellier, 27-29 octobre 2004.

PANDEY A., « Greening Garhwal through stakeholder engagement: the role of ecofeminism, community and the state in sustainable development», Sustainable Development, 18(1), p. 12-19, janv-fév, 2010.

PARADAS A., «Développement durable en petites entreprises. De la sensibilisation à l'engagement », La Revue des Sciences de Gestion, Direction et Gestion, n 247-248, janvier-avril, p. 129-137, 2011. 
RousseAu D., «L'avenir économique appartient aux valeurs féminines », Clés, $\quad$ http://www.cles.com/entretiens/article/l-avenir-economiqueappartient-aux\#, 2007.

Revue industrie, "Chef d'entreprise au féminin », $\mathrm{n}^{\circ} 128$, Ministère de l'Economie, des Finances et de l'Emploi, 14 janvier, 2008. http://www.Industrie.gouv.fr/biblioth/docu/kiosque/cahiers/c128p2627.html.

St-Pierre J., Carrier C., Pilaeva K., « Développement durable et PME : les femmes ont-elles une conception différente de celle des hommes»? Colloque International : PME en marche vers le développement durable, Montréal, Octobre, 2011.

Vidal C., «Nos cerveaux : tous pareils, tous différents », Conférence, les Conférences de la Cité, Cité des Sciences, 8 mars 2006. Féminin/masculin : mythes scientifiques et idéologie, sous la direction de Catherine Vidal, Editions Belin, mars 2006.

Vier MACHADO H., Rouleau L., «Identité sociale et entrepreneuriat féminin: étude comparative entre le Brésil et le Canada », $6^{\circ}$ Congrès international francophone sur la PME, HEC Montréal, Oct, 2002.

Zouiten J., LEVY TADJine T., «Les femmes et les immigrés ont-ils besoin d'un accompagnement entrepreneurial spécifique? "IVè Congrès de l'Académie de l'Entrepreneuriat, Paris, 24-25 nov 2005.

Zuinen N., Essai sur le rôle des femmes et des valeurs féminines, Reflets et perspectives de la vie économique, Vol 1 tome XLI, p. 109-114, De Boeck Université, 2002. 


\section{Annexe 1}

Tableau 1 : Répartition des modalités contributives selon les deux premiers axes ${ }^{8}$

\begin{tabular}{|c|c|c|c|c|}
\hline \multirow[b]{2}{*}{$\begin{array}{l}\text { Variables et } \\
\text { modalités }\end{array}$} & \multicolumn{2}{|l|}{ Axe $1(44.36 \%)$} & \multicolumn{2}{|l|}{ Axe $2(16.79 \%)$} \\
\hline & Coordonnées + & Coordonnées - & Coordonnées + & Coordonnées - \\
\hline \multicolumn{5}{|l|}{ Env. } \\
\hline $21 / 1$ & & & Tri déchets - & \\
\hline $21 / 2$ & $\begin{array}{l}\text { Réutilisation } \\
\text { déchets? }\end{array}$ & $\begin{array}{l}\text { Réutilisation } \\
\text { déchets - }\end{array}$ & & $\begin{array}{l}\text { Réutilisation } \\
\text { déchets = }\end{array}$ \\
\hline $21 / 3$ & $\begin{array}{l}\text { Valorisation } \\
\text { déchets? }\end{array}$ & & $\begin{array}{l}\text { Valorisation } \\
\text { déchets - }\end{array}$ & $\begin{array}{l}\text { Valorisation } \\
\text { déchets + }\end{array}$ \\
\hline $21 / 4$ & $\begin{array}{l}\text { Energies } \\
\text { renouvelables? }\end{array}$ & & & $\begin{array}{l}\text { Energies } \\
\text { renouvelables }+ \text { et } \\
=\end{array}$ \\
\hline $21 / 5$ & $\begin{array}{l}\text { Production non } \\
\text { polluante? }\end{array}$ & & $\begin{array}{l}\text { Production non } \\
\text { polluante? }\end{array}$ & $\begin{array}{l}\begin{array}{l}\text { Production non } \\
\text { polluante }=\end{array} \\
\end{array}$ \\
\hline $21 / 6$ & & $\begin{array}{l}\text { Economie eau } \\
\text { énergie - }\end{array}$ & & \\
\hline $21 / 7$ & Eco-conception? & & & \\
\hline $21 / 8$ & Réseaux env. ? & Réseaux env. - & & Réseaux env. = \\
\hline \multicolumn{5}{|l|}{ Social } \\
\hline $29 / 1$ & $\begin{array}{l}\text { Investissement } \\
\text { formation sup? }\end{array}$ & $\begin{array}{l}\text { Investissement } \\
\text { formation sup - }\end{array}$ & $\begin{array}{l}\text { Investissement } \\
\text { formation sup ? et }\end{array}$ & $\begin{array}{l}\text { Investissement } \\
\text { formation sup = et } \\
+\end{array}$ \\
\hline $29 / 2$ & $\begin{array}{l}\text { Formation } \\
\text { compétences? }\end{array}$ & $\begin{array}{l}\text { Formation } \\
\text { compétences - }\end{array}$ & $\begin{array}{l}\text { Formation } \\
\text { compétences? et - }\end{array}$ & $\begin{array}{l}\text { Formation } \\
\text { compétences }=\text { et } \\
+\end{array}$ \\
\hline $29 / 3$ & Sécurité travail ? & Sécurité travail - & $\begin{array}{l}\text { Sécurité travail ? } \\
\text { et- }\end{array}$ & Sécurité travail + \\
\hline $29 / 4$ & $\begin{array}{l}\text { Amélioration } \\
\text { conditions W ? }\end{array}$ & $\begin{array}{l}\text { Amélioration } \\
\text { conditions W. }\end{array}$ & $\begin{array}{l}\text { Amelioration } \\
\text { conditions } W \text { ? et }\end{array}$ & $\begin{array}{l}\text { Amélioration } \\
\text { conditions } \mathrm{W}=\end{array}$ \\
\hline $29 / 5$ & $\begin{array}{l}\text { Recrutement } \\
\text { diversité âges? }\end{array}$ & $\begin{array}{l}\text { Recrutement } \\
\text { diversité âges = }\end{array}$ & $\begin{array}{l}\text { Recrutement } \\
\text { diversité âges? }\end{array}$ & $\begin{array}{l}\text { Recrutement } \\
\text { diversité âges + }\end{array}$ \\
\hline $29 / 6$ & Parité HF? & & & Parité HF + \\
\hline $29 / 7$ & Handicap ? et + & Handicap- & Handicap - & Handicap $=\mathrm{et}+$ \\
\hline $29 / 8$ & $\begin{array}{l}\text { Participation } \\
\text { bénéfices? }\end{array}$ & $\begin{array}{l}\text { Participation } \\
\text { bénef- }\end{array}$ & $\begin{array}{l}\text { Participation } \\
\text { bénéfices? et - }\end{array}$ & $\begin{array}{l}\text { Participation } \\
\text { bénéfices + }\end{array}$ \\
\hline $29 / 9$ & Réseaux GRH ? & Réseaux GRH - & Réseaux GRH ? & $\begin{array}{l}\text { Réseaux GRH = et } \\
+\end{array}$ \\
\hline \multicolumn{5}{|l|}{ Sociétal } \\
\hline $37 / 1$ & & & & $\begin{array}{l}\text { Recrutement local } \\
=\end{array}$ \\
\hline $37 / 2$ & $\begin{array}{l}\text { Approvisionnement } \\
\text { local? }\end{array}$ & $\begin{array}{l}\text { Approvisionneme } \\
\text { nt local }+\end{array}$ & & \\
\hline $37 / 3$ & Partenariats? & Partenariats - & & Partenariats $=$ et + \\
\hline $37 / 4$ & $\begin{array}{l}\text { Philanthropie } \\
\text { mécénat? }\end{array}$ & $\begin{array}{l}\text { Philanthropie } \\
\text { mécénat - }\end{array}$ & & \\
\hline $37 / 5$ & Ecoute riverains? & & & Ecoute riverains $=$ \\
\hline \multirow[t]{3}{*}{$37 / 6$} & Réseaux insertion? & Réseaux insertion & & $\begin{array}{l}\text { Réseaux } \\
\text { insertion }=\end{array}$ \\
\hline & Hommes & Femmes & Hommes & Femmes \\
\hline & \multicolumn{2}{|c|}{$\begin{array}{l}\text { L'axe } 1 \text { met en évidence une opposition } \\
\text { générale entre les "non-réponses" (plus } \\
\text { proches des coordonnées hommes) et un } \\
\text { nombre important de réponses négatives } \\
\text { sauf } 2 \text { (femmes). }\end{array}$} & \multicolumn{2}{|c|}{$\begin{array}{l}\text { L'axe } 2 \text { met en évidence une opposition } \\
\text { entre des modalités - et? (proches } \\
\text { coordonnées hommes) et des modalités + } \\
\text { et }=(\text { femmes). }\end{array}$} \\
\hline
\end{tabular}

8. Les modalités surlignées en rouge correspondent aux réponses négatives (-) Les modalités surlignées en vert correspondent aux réponses positives $(+)$ Les modalités surlignées en beige correspondent aux réponses neutres $(=)$ Les modalités surlignées en gris correspondent aux non-réponses ( ?) 


\section{Annexe 2}

Tableau 3 - Niveau de pratiques RSE selon le genre des entrepreneurs $(\min =0 ; \max =5)$. Tests paramétriques $\mathrm{T}$ de Student

\begin{tabular}{|c|c|c|c|c|c|}
\hline Pratiques & Natures de la pratique & Femme & Homme & $\begin{array}{l}\text { Valeur } \\
\mathrm{T}\end{array}$ & $\begin{array}{l}\text { Valeur } \\
\mathrm{U}\end{array}$ \\
\hline \multirow{8}{*}{$\begin{array}{l}\text { Environne } \\
\text { mentales }\end{array}$} & Tri des déchets & 3,74 & 3,48 & 0,540 & 295,5 \\
\hline & Réutilisation des déchets & 1,65 & 1,48 & 0,389 & 285,5 \\
\hline & Valorisation des déchets & 2,78 & 2,35 & 0,727 & 285,5 \\
\hline & $\begin{array}{l}\text { Utilisation des énergies } \\
\text { renouvelables }\end{array}$ & 1,83 & 1,52 & 0,612 & 276,0 \\
\hline & $\begin{array}{l}\text { Méthodes de production peu } \\
\text { ou pas polluantes }\end{array}$ & 2,13 & 1,43 & 1,404 & 325,0 \\
\hline & Économie Eau/Énergie & 2,87 & 3,52 & $-0,652$ & 201,0 \\
\hline & $\begin{array}{l}\text { Ecoconception (prise en } \\
\text { compte de l'impact des } \\
\text { produits sur l'environnement) }\end{array}$ & 3,17 & 2,30 & $1,652^{*}$ & $341,5^{*}$ \\
\hline & $\begin{array}{l}\text { Participation à des réseaux } \\
\text { professionnels } \\
\text { environnementaux }\end{array}$ & 1,91 & 1,61 & 0,632 & 280,5 \\
\hline \multirow{9}{*}{ Sociales } & $\begin{array}{l}\text { Investissement dans la } \\
\text { formation personnel }>\text { loi }\end{array}$ & 2,83 & 2,39 & 0,938 & 304,0 \\
\hline & $\begin{array}{ll}\text { Investissement dans la } \\
\text { formation personnel } \\
\text { compétences nécessaires }\end{array}$ & 2,57 & 2,35 & 0,502 & 278,0 \\
\hline & $\begin{array}{l}\text { Investissement dans la sécurité } \\
\text { au travail > loi }\end{array}$ & 3,00 & 2,74 & 0,564 & 290,0 \\
\hline & $\begin{array}{l}\text { Modification organisation du } \\
\text { travail pour améliorer les } \\
\text { conditions de travail }\end{array}$ & 3,26 & 3,09 & 0,391 & 266,0 \\
\hline & Recrutement salarié tout âge & 3,13 & 3,09 & 0,084 & 276,5 \\
\hline & $\begin{array}{l}\text { Assurer la parité hommes / } \\
\text { femmes }\end{array}$ & 2,39 & 2,57 & $-0,353$ & 250,0 \\
\hline & $\begin{array}{l}\text { Recrutement de salariés } \\
\text { handicapés }\end{array}$ & 1,39 & 2,13 & $\overline{1}, 792^{*}$ & 207,0 \\
\hline & $\begin{array}{l}\text { Participation et intéressement } \\
\text { des salariés aux bénéfices de } \\
\text { l'entreprise }\end{array}$ & 2,52 & 2,09 & 0,828 & 308,0 \\
\hline & $\begin{array}{l}\text { Participation à des réseaux } \\
\text { professionnels sociaux et GRH }\end{array}$ & 1,43 & 1,65 & $-0,507$ & 245,5 \\
\hline \multirow{6}{*}{$\begin{array}{l}\text { Sociétales } \\
\text { ou } \\
\text { communautaires }\end{array}$} & Recrutement local ou régional & 3,96 & 4,30 & $-0,923$ & 232,0 \\
\hline & $\begin{array}{l}\text { Approvisionnement local ou } \\
\text { régional }\end{array}$ & 3,35 & 2,65 & 1,381 & 320,0 \\
\hline & $\begin{array}{l}\text { Partenariats avec des ONG, } \\
\text { associations ou universités }\end{array}$ & 1,39 & 1,52 & $-0,338$ & 255,5 \\
\hline & $\begin{array}{l}\text { Investissements dans des } \\
\text { actions philanthropiques, } \\
\text { mécénat, sponsoring }\end{array}$ & 1,78 & 1,57 & 0,548 & 302,0 \\
\hline & $\begin{array}{l}\text { Prise en compte des riverains } \\
\text { et de leurs réclamations }\end{array}$ & 2,61 & 2,48 & 0,222 & 283,0 \\
\hline & $\begin{array}{l}\text { Participation à des réseaux } \\
\text { professionnels d'insertion dans } \\
\text { la société }\end{array}$ & 1,91 & 1,96 & $-0,094$ & 269,0 \\
\hline
\end{tabular}

